\title{
DISCLAIMER
}

WSRC-TR--91-474-3

This report was prepared as an account of work sponsored by an agency of the United States Government. Neither the United States Government nor any agency thereof, nor any of their employees, makes any warranty, express or implied, or assumes any legal liability or responsibility for the accuracy, completeness, or usefulness of any information, apparatus, product, or process disclosed, or represents that its use would not infringe privately owned rights. Reference herein to any specific commercial product, process, or service by trade name, trademark, manufacturer, or otherwise does not necessarily constitute or imply its endorsement, recommendation, or favoring by the United States Government or any agency thereof. The views and opinions of authors expressed herein do not necessarily state or reflect those of the United States Government or any agency thereof.

\section{F/H AREA ETF EFFLUENT (H-016 OUTFALL) CERIODAPHNIA SURVIVAL/REPRODUCTION TEST, TEST DATE: JUNE 27, 1991 (U)}

by

W. L. Specht, et al.

Westinghouse Savannah River Company

Savannah River Laboratory

Aiken, South Carolina 29808

A Technical R`sport for Publication

$$
\text { ando when }
$$

This paper was prepared in connection with work done under Contract No. DE-AC09-89SR18035 with the U.S. Department of Energy. By acceptance of this paper, the publisher and/or recipient acknowledges the U.S. Government's right to retain a nonexclusive, royalty-free license in and to any copyright covering this paper, along with the right to reproduce and to authorize others to reproduce all or part of the copyrighted paper. 
Derivative Classifier Domereshener

D. B. Moore-Shedrow, Section Manager

Authorized Derivative Classifer

F/H AREA ETF EFFLUENT (H.016 OUTFALL)

CERIODAPHNIA SURVIVAL/REPRODUCTION TEST,

TEST DATE: JUNE 27, 1991 (U)

WSRC Technical Representative: W. L. Specht

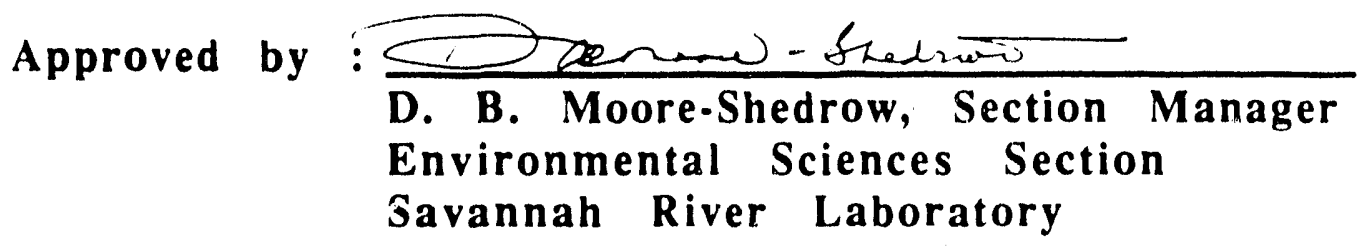

Publication Date: August 1991

WESTINGHOUSE SAVANNAH RIVER COMPANY

SAVANNAH RIVER SITE

AIKEN, SC 29808

Prepared for the U.S. Department of Energy under Contract No. DE-AC09-88SR18035 
4. Chem-Nuclear Laboratory Services, Inc.

Posi Office Box 17467

Greenville South Carolina 29606

(803) 235-j;07

\section{Ceriedaphnia SURVIVAL/REPRODOCTION TEST}

Sample Identification: H - F Area (U3RC)

Test Date: June 27, 1991

Client: Savannah River Site

Location: Aiken, South Carolina 


\section{INTERPREIATIOW OF RBSULTS}

This toxicity test was conducted to determine if the effluent affects the survival or reproduction of the test organisms during a seven day period. The test involved exposing the test organisms to a series of dilutions of the effluent. At each dilution the survival and reproduction of ten test organisms was recorded. Each effluent dilution was compared to a control set of test organisms. Survival data were analyzed by Fisher's Exact Test and Probit Analysis (or Trimed spearman Karber if Probit can not be used) to determine the effluent concentration necessary to cause statistically significant ( $p=0.05)$ mortality. Reproduction data was analyzed for normality, homogeneity of variance and equality of replicates among dilutions to determine the appropriate statistical test for analysis of statistical differences among dilutions. Results are summarized as follows:

Acute Toxicity:

Trimed Spearman-Karber

$$
\text { LC50 }=54.77 \text { Effluent }
$$

Fisher's Exact Test

$$
\begin{array}{rr}
\text { NOEC }= & 30.08 \text { Effluent } \\
\text { LOEC }= & 100.08 \text { Effluent } \\
\text { ChV }= & 54.778 \text { Effluent }
\end{array}
$$

Chronic Toxicity

T-test with Bonferroni adjustment

(NOEC) No Observed Effect Conc.: 


\section{SAYPITE InTORTATIOA}

Three effluent samples were collected by and transported to Chem-Nuclear Laboratory Services by on-site personnel. Samples were collected in four one liter plastic cubitainers.

\section{Client: Savannah River Site \\ Sample Identification: H - F Area (U3RC) \\ Start Date: June 27, 1991}

$\begin{array}{lll}\text { Sample } & \text { Sample } & \text { Sample } \\ \$ 2 & \$ 3\end{array}$

\section{Lab \\ Type \\ Date sampled \\ Time sampled}

Days of use

Maximum hold time:

Temperature Upon Receipt:

$$
\begin{array}{rrr}
\text { BI0871-1 } & \text { BI0871-2 } & \text { BIO871-3 } \\
\text { grab } & \text { grab } & \text { grab }
\end{array}
$$

$6 / 26 / 916 / 28 / 91 \quad 7 / 01 / 91$

$9: 55$ AM $9: 30$ AM $9: 30 \mathrm{AM}$
0,1
2,3
$4,5,6$

54 hrs 58 hrs 55 hrs

$3.0^{\circ} \mathrm{C} \quad 3.0^{\circ} \mathrm{C} \quad 8.0^{\circ} \mathrm{C}$

EFFLUENT FIELD PARAMETERS

Sample $11 \quad$ Sample 2 Sample 3

$\begin{array}{lccc}\text { D.O. }(\mathrm{mg} / \mathrm{L}) & 6.5 & 7.2 & 7.3 \\ \mathrm{pH} & 6.95 & 6.9 & 7.33 \\ \text { Temp }(\mathrm{C}) & 21.0^{\circ} \mathrm{C} & 25.8^{\circ} \mathrm{C} & 27.0^{\circ} \mathrm{C} \\ \text { Res Cl }(\mathrm{mg} / \mathrm{L}) & <.05 & <.05 & <.05\end{array}$


Client: Savannah River site

Sriple Identification: H - F Area (U3RC)

Stot Date: June 27, 1991

Tux Type: 7 Day Chronic Toxicity - Reproduction Test

T* Location: Chem-Nuclear Laboratory Services

Text organism: ceriodaphnia dubia

Source: $\quad$ CNLS

Age: $\quad<24$ hrs

'Tect Procedure: Short-Term Methods for Estimating the Chronic Toxicity of Effluents and Receiving Waters to Freshwater organisms. 2nd Edition. EPA 600/4-85/014 Method 1002.0

Tut vessels: $\quad 30 \mathrm{ml}$ plastic beakers

Amoant Test Soln: $15 \mathrm{ml} /$ replicate

Deplicates/Concentration: 10 (test vessels randomized)

Tet Organisms/Replicate: 1

\# Test Concentrations Used (excluding control):

Te: Solution Renewal: Daily

Food Source: $\quad 0.10 \mathrm{ml}$ Selenastrum $\left(3.1-3.5 \times 10^{7} \mathrm{cells} / \mathrm{ml}\right)$ and $0.05 \mathrm{ml}$ YCT daily / test vessel

Test ing Performed, Results Checked and ieport Prepared BY:
Joel stephens

Analytical Biologist

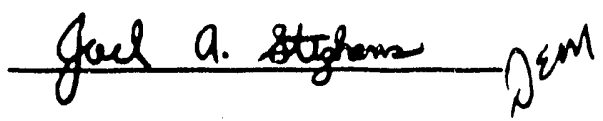




\section{RESUITS}

Client: Savannah River site

Sample Identification: H - F Area (U3RC)

Start Date: June 27, 1991

SURVIVAL DATA (At Test Termination)

\begin{tabular}{|c|c|c|c|c|c|c|}
\hline & 0.08 & 18 & $\begin{array}{r}\text { Eff } \\
38\end{array}$ & $\begin{array}{l}\text { Conc } \\
108\end{array}$ & $\begin{array}{c}\text { ration } \\
308\end{array}$ & $100 \%$ \\
\hline Survival & $\begin{array}{c}10 \\
1008\end{array}$ & $\begin{array}{c}10 \\
1008\end{array}$ & $\begin{array}{c}10 \\
1008\end{array}$ & $\begin{array}{l}10 \\
1008\end{array}$ & $\begin{array}{l}10 \\
1008\end{array}$ & $\begin{array}{l}0 \\
08\end{array}$ \\
\hline $\begin{array}{r}\text { Mortality } \\
(7 \text { day })\end{array}$ & $\begin{array}{l}0 \\
08\end{array}$ & $\begin{array}{l}0 \\
08\end{array}$ & $\begin{array}{l}0 \\
0 \text { \& }\end{array}$ & $\begin{array}{l}0 \\
08\end{array}$ & $\begin{array}{l}0 \\
08\end{array}$ & $\begin{array}{c}10 \\
100 \%\end{array}$ \\
\hline $\begin{array}{l}\text { Lost } \\
\text { Males } \\
\text { Bad Brood }\end{array}$ & $\begin{array}{l}0 \\
0 \\
0\end{array}$ & $\begin{array}{l}1 \\
1 \\
0\end{array}$ & $\begin{array}{l}0 \\
0 \\
0\end{array}$ & $\begin{array}{l}0 \\
0 \\
0\end{array}$ & $\begin{array}{l}1 \\
0 \\
0\end{array}$ & $\begin{array}{l}0 \\
0 \\
0\end{array}$ \\
\hline
\end{tabular}

SURVIVAL - DATA ANALYSIS

Probit Analysis

LC5O $=54.77$ \&ffluent

Fisher's Exact Test

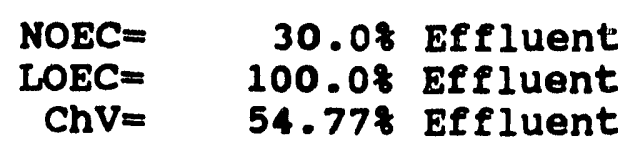

BIO - 871 


\section{RRSURTS}

ilient: Savannah River Site

janple Identification: $H-F$ Area (U3RC)

jtart Date: June 27, 1991

:HRONIC TOXICITY - Daily Reproduction Totals

\begin{tabular}{|c|c|c|c|c|c|c|}
\hline & Rep & Day 4 & Day 5 & Day 6 & Day 7 & Total \\
\hline Control & $\begin{array}{r}\text { A } \\
\text { B } \\
\text { C } \\
\text { D } \\
\text { E } \\
\text { F } \\
\text { G } \\
\text { H } \\
\text { I } \\
\text { J } \\
\text { Total }\end{array}$ & $\begin{array}{r}1 \\
5 \\
3 \\
1 \\
5 \\
1 \\
1 \\
11 \\
0 \\
4 \\
32\end{array}$ & $\begin{array}{r}9 \\
3 \\
2 \\
2 \\
6 \\
4 \\
4 \\
0 \\
3 \\
19 \\
52\end{array}$ & $\begin{array}{r}12 \\
8 \\
13 \\
8 \\
10 \\
12 \\
12 \\
10 \\
8 \\
0 \\
93\end{array}$ & $\begin{array}{l}0 \\
0 \\
0 \\
0 \\
0 \\
1 \\
7 \\
1 \\
7 \\
15 \\
31\end{array}$ & $\begin{array}{l}22 \\
16 \\
18 \\
11 \\
21 \\
18 \\
24 \\
22 \\
18 \\
38 \\
208\end{array}$ \\
\hline$\frac{18}{\text { Effluent }}$ & $\begin{array}{r}\text { A } \\
\text { B } \\
\text { C } \\
\text { D } \\
\text { E } \\
\text { F } \\
\text { G } \\
\text { H } \\
\text { I } \\
\text { J }\end{array}$ & $\begin{array}{l}0 \\
7 \\
0 \\
2 \\
M \\
1 \\
0 \\
5 \\
5 \\
4 \\
24\end{array}$ & $\begin{array}{l}0 \\
8 \\
4 \\
9 \\
M \\
4 \\
9 \\
6 \\
L \\
6 \\
46\end{array}$ & $\begin{array}{r}13 \\
0 \\
10 \\
0 \\
M \\
10 \\
7 \\
17 \\
2 \\
4 \\
61\end{array}$ & $\begin{array}{r}16 \\
8 \\
7 \\
13 \\
4 \\
8 \\
0 \\
0 \\
L \\
0 \\
52\end{array}$ & $\begin{array}{r}29 \\
23 \\
21 \\
24 \\
0 \\
23 \\
16 \\
28 \\
5 \\
14 \\
178\end{array}$ \\
\hline $\begin{array}{r}3.08 \\
\text { Effluent }\end{array}$ & $\begin{array}{r}\text { A } \\
\text { B } \\
\text { C } \\
\text { D } \\
\text { E } \\
\text { F } \\
\text { G } \\
\text { H } \\
\text { I } \\
\text { J }\end{array}$ & $\begin{array}{r}4 \\
5 \\
0 \\
5 \\
7 \\
1 \\
0 \\
0 \\
6 \\
0 \\
28\end{array}$ & $\begin{array}{r}7 \\
8 \\
3 \\
10 \\
0 \\
8 \\
13 \\
13 \\
8 \\
10 \\
80\end{array}$ & $\begin{array}{r}14 \\
0 \\
5 \\
0 \\
10 \\
16 \\
16 \\
0 \\
13 \\
17 \\
91\end{array}$ & $\begin{array}{r}0 \\
11 \\
4 \\
12 \\
9 \\
0 \\
0 \\
7 \\
0 \\
0 \\
43\end{array}$ & $\begin{array}{r}25 \\
24 \\
12 \\
27 \\
26 \\
25 \\
29 \\
20 \\
27 \\
27 \\
242\end{array}$ \\
\hline
\end{tabular}




\section{RRSULIS}

Client: Savannah River site

Sample Identification: H - F Area (U3RC)

Start Date: June 27, 1991

CHRONIC TOXICITY - Daily Reproduction Totals

\begin{tabular}{|c|c|c|c|c|c|c|}
\hline & Rep & Day 4 & Day 5 & Day 6 & Day 7 & Total \\
\hline $\begin{array}{r}10.08 \\
\text { Effluent }\end{array}$ & $\begin{array}{r}\text { A } \\
\text { B } \\
\text { C } \\
\text { D } \\
\text { E } \\
\text { F } \\
\text { G } \\
\text { H } \\
\text { I } \\
\text { J } \\
\text { Total }\end{array}$ & $\begin{array}{r}6 \\
0 \\
0 \\
2 \\
3 \\
0 \\
3 \\
0 \\
2 \\
0 \\
16\end{array}$ & $\begin{array}{r}3 \\
8 \\
10 \\
0 \\
4 \\
10 \\
2 \\
0 \\
13 \\
5 \\
55\end{array}$ & $\begin{array}{r}10 \\
9 \\
12 \\
12 \\
0 \\
15 \\
12 \\
0 \\
0 \\
10 \\
80\end{array}$ & $\begin{array}{r}12 \\
0 \\
0 \\
5 \\
10 \\
0 \\
5 \\
4 \\
11 \\
1 \\
48\end{array}$ & $\begin{array}{r}31 \\
17 \\
22 \\
19 \\
17 \\
25 \\
22 \\
4 \\
26 \\
16 \\
199\end{array}$ \\
\hline $\begin{array}{r}30.0 \% \\
\text { Effluent }\end{array}$ & $\begin{array}{r}\text { A } \\
\text { B } \\
\text { C } \\
\text { D } \\
\text { E } \\
\mathbf{F} \\
\mathbf{G} \\
\text { H } \\
\text { I } \\
\text { J }\end{array}$ & $\begin{array}{r}5 \\
6 \\
L \\
4 \\
5 \\
0 \\
0 \\
0 \\
4 \\
4 \\
28\end{array}$ & $\begin{array}{l}0 \\
8 \\
L \\
0 \\
7 \\
0 \\
0 \\
0 \\
8 \\
6 \\
29\end{array}$ & $\begin{array}{r}7 \\
0 \\
1 \\
12 \\
0 \\
6 \\
0 \\
1 \\
0 \\
1 \\
27\end{array}$ & $\begin{array}{r}12 \\
7 \\
L \\
11 \\
12 \\
0 \\
9 \\
0 \\
6 \\
8 \\
65\end{array}$ & $\begin{array}{r}24 \\
21 \\
0 \\
27 \\
24 \\
6 \\
9 \\
1 \\
18 \\
19 \\
149\end{array}$ \\
\hline $\begin{array}{r}100.0 \% \\
\text { Effluent }\end{array}$ & $\begin{array}{r}\text { A } \\
\text { B } \\
\text { C } \\
\text { D } \\
\text { E } \\
\text { F } \\
\text { G } \\
\text { H } \\
\text { I } \\
\text { J } \\
\text { Total }\end{array}$ & $\begin{array}{l}\mathbf{X} \\
\mathbf{X} \\
\mathbf{X} \\
\mathbf{X} \\
\mathbf{X} \\
\mathbf{X} \\
\mathbf{X} \\
\mathbf{X} \\
\mathbf{x} \\
\mathbf{X} \\
\mathbf{0}\end{array}$ & $\begin{array}{l}\mathbf{X} \\
\mathbf{X} \\
\mathbf{X} \\
\mathbf{X} \\
\mathbf{X} \\
\mathbf{X} \\
\mathbf{X} \\
\mathbf{X} \\
\mathbf{X} \\
\mathbf{X} \\
\mathbf{0}\end{array}$ & $\begin{array}{l}\mathbf{X} \\
\mathbf{X} \\
\mathbf{X} \\
\mathbf{X} \\
\mathbf{X} \\
\mathbf{X} \\
\mathbf{X} \\
\mathbf{X} \\
\mathbf{X} \\
\mathbf{X} \\
\mathbf{0}\end{array}$ & $\begin{array}{l}\mathbf{X} \\
\mathbf{X} \\
\mathbf{X} \\
\mathbf{X} \\
\mathbf{X} \\
\mathbf{X} \\
\mathbf{X} \\
\mathrm{X} \\
\mathbf{X} \\
\mathbf{X} \\
\mathbf{0}\end{array}$ & $\begin{array}{l}0 \\
0 \\
0 \\
0 \\
0 \\
0 \\
0 \\
0 \\
0 \\
0 \\
0\end{array}$ \\
\hline
\end{tabular}

$\mathrm{X}$ represents the mortality of the test organism.

BIO - 871 


\section{RRSULTS}

Client: Savannah River Site

Sample Identification: H-F Area (U3RC)

Start Date: June 27, 1991

CHRONIC

TOXICITY

- Statistical Analysis

T-test with Bonferroni adjustment

(Assumes Data is Normally Distributed and Homogeneous)

$\begin{array}{lllllll}\text { Conc. } & 0.08 & 18 & 3.08 & 10.08 & 30.1\end{array}$

Average Young.

per Fenale

20.8

22.3

24.2

19.9

16.6

std. Dev.

7.08

5.23

4.92

7.31

9.07

Calculated Value

$-.443-1.102$

0.292

1.339

Critical Value

2.329

2.329

2.329

2.329

Reproduction

no

no

no

no

significantly less

than control?

( 958 confidence)

\section{8 conf.}

(NOEC) No Observed Effect Conc.: $30.0 \%$

(LOEC) Lowest Observed Effect Conc.: 100.08

Chronic Value: $\quad 54.778$

The data are normally distributed.

The data are homogeneous in variance.

The number of replicates in each concentration is nst equal.

The appropriate statistical test is the T-test with

Bonferroni adjustment. 
Client: Savannan River site

Sample Identification: H-F Area (U3RC)

Start Date: June 27, 1991

Dllution Water

ID: Upper Three Runs Creek Water

Preparation: Filtered through a 37 micron Nitex mesh.

Temperature Heasurements ( $\left.{ }^{\circ} \mathrm{C}\right)$

\begin{tabular}{rrrrrrrr}
\hline Day 0 & Day 1 & Day 2 & Day 3 & Day 4 Day 5 Day 6 & Day 7 \\
25.2 & 26.0 & 26.0 & 27.7 & 26.4 & 26.4 & 25.7 & 24.9
\end{tabular}

Effluent Chenietry Parameters

Conductivity

(umhos)

Sample 1

Sample 2

Sample 3
Hardness

$(m g / L)$

$<1.0$

$<1.0$

1.0
Alkalindty

$(\mathrm{mg} / \mathrm{L})$

24.0

16.2

21.2
Res. Cl. $(m g / L)$

$<0.05$

$<0.05$

$<0.05$ 
WATER CHEYISTRY RMAXSIS

ient: Savannah RIver Site
mple Identification: H Area (U3RC)
art Date: June 27,1991

st Solutions

NTROL

pH D.O. D.O. Hardness Alkalinity Conduc.

$(\mathrm{mg} / \mathrm{L}) \quad\left(\begin{array}{lll}\mathrm{f}\end{array}\right) \quad(\mathrm{mg} / \mathrm{L}) \quad(\mathrm{mg} / \mathrm{L}) \quad$ (umhos)

itial

Y 1 (old)

6.00

$y 1$ (new)

6.60

5.90

$y 2$ (old)

6.20

y 2 (new)

6.40

Y 3 (old)

6.30

y 3 (new)

5.80

Y 4 (old)

6.00

y 4 (new)

6.40

6.80

j 5 (old)

$\begin{array}{lll}Y & 5 & \text { (new) } \\ y & 6 & (01 d)\end{array}$

6.30

6.80

y 6 (new)

6.40

nal

6.80

\section{2}

8.0

8.0

4.21

21

8.2

8.0

5.26

21

8.3

8.3

7.0

7.5

8.0

5.26

20

9.0

10.0

5.26

22

8.1

6.3

7.2

5.26

19

8.1

8.3

5.26

19

928

8.2

1058

$101 \%$

7.0

4.21

17

Effluent

$\begin{array}{cccc}\text { D.O. } & \text { D.O } & \text { pH } & \begin{array}{c}\text { Conductivity } \\ \text { (umhos) }\end{array}\end{array}$

\section{itial}

y 1 (old)

$y 1$ (new)

iy 2 (old)

y 2 (new)

y 3 (old)

y 3 (new)

iy 4 (old)

:y 4 (new)

1y 5 (old)

y 5 (new)

iy 6 (old)

1y 6 (new)

nal

$\begin{array}{rrrr}8.2 & 1038 & 6.00 & 23 \\ 8.0 & 1038 & 6.70 & \\ 8.1 & 1048 & 5.90 & 23 \\ 8.2 & 1058 & 6.30 & \\ 7.9 & 1018 & 6.40 & 23 \\ 6.9 & 888 & 6.40 & \\ 7.3 & 948 & 5.90 & 23 \\ 7.9 & 1018 & 6.10 & \\ 8.1 & 1048 & 6.30 & 21 \\ 6.4 & 828 & 6.90 & \\ 7.1 & 918 & 6.30 & 20 \\ 8.0 & 1018 & 6.80 & \\ 8.2 & 1048 & 6.40 & 19 \\ 8.2 & 1018 & 6.80 & \end{array}$




\section{WATIER CHESTSYRY ANAIYSIS}

Client: Savannah River Site

Sample Identification:

H - F Area (U3RC)

Start Date: June 27, 1991

3.08 effluent

\section{Initial}

Day 1 (old)

Day 1 (new)

Day 2 (old)

Day 2 (new)

Day 3 (old)

Day 3 (new)

Day 4 (old)

Day 4 (new)

Day 5 (old)

Day 5 (new)

Day 6 (old)

Day 6 (new)

Final

\begin{tabular}{|c|c|c|c|}
\hline $\begin{array}{l}D .0 \\
(\mathrm{mg} / \mathrm{L})\end{array}$ & $\left(\begin{array}{c}0.0 \\
(q)\end{array}\right.$ & $\mathrm{pH}$ & $\begin{array}{c}\text { Conductivity } \\
\text { (umhos) }\end{array}$ \\
\hline
\end{tabular}

$\begin{array}{llll}8.3 & 104 \% & 6.10 & 26\end{array}$

$8.2 \quad 1058 \quad 6.80$

$8.2 \quad 1058 \quad 5.90$

$7.9 \quad 1018 \quad 6.40$

$8.1 \quad 1048 \quad 6.60$

$7.2 \quad 928 \quad 6.40$

$\begin{array}{lll}7.1 & 918 & 6.00\end{array}$

$8.0 \quad 1038 \quad 6.20$

$8.2658 \quad 6.40$

$\begin{array}{lll}6.5 & 838 & 7.00\end{array}$

$7.1 \quad 918 \quad 6.30$

$8.2 \quad 1048 \quad 6.90$

$8.2 \quad 1048 \quad 6.40$

$8.2 \quad 1018 \quad 6.90$
26

26

25

25

23

21

21

\subsection{8 effluent}

Initial

Day 1 (old)

Day 1 (new)

Day 2 (old)

Day 2 (new)

Day 3 (old)

Day 3 (new)

Day 4 (old)

Day 4 (new)

Day 5 (old)

Day 5 (new)

Day 6 (old)

Day 6 (new)

Final

\begin{tabular}{|c|c|c|c|}
\hline $\begin{array}{l}D .0 \\
(m g / L)\end{array}$ & $(i)$ & $\mathrm{pH}$ & $\begin{array}{c}\text { Conductivity } \\
\text { (umhos) }\end{array}$ \\
\hline
\end{tabular}

$8.3 \quad 1047 \quad 6.20$

45

$8.3 \quad 1068 \quad 6.90$

$8.265 \% 6.00$

$8.0 \quad 103 \% \quad 6.50$

$8.2658 \quad 105860$

$7.1 \quad 918 \quad 6.50$

$7.0 \quad 908 \quad 6.10$

$8.1 \quad 1048 \quad 6.30$

$8.1 \quad 1048 \quad 6.40$

$\begin{array}{lll}6.6 & 858 & 7.00\end{array}$

$7.1 \quad 918 \quad 6.50$

$8.1 \quad 1038 \quad 7.00$

$8.2 \quad 1048 \quad 6.50$

$8.3 \quad 6.90$ 
Client: Savannah River site

Sample Identification: H - F Area (U3RC)

Start Date: June 27, 1991

30\% Effluent

D.O.

$(\mathrm{mg} / \mathrm{L})$
$\mathrm{pH}$

$\left(\begin{array}{c}0 \\ (8)\end{array}\right)$

1038

1058

$105 \%$

1038

$104 \%$

928

888

$104 \%$

1048

838

91.8

1038

$104 t$

$101 z$
Hardness

$(\mathrm{mg} / \mathrm{L})$
Alkalinity ( $\mathrm{mg} / \mathrm{L})$
Conduc

(umhos)

Day 6 (new)

Final

6.30

7.20

6.20

6.70

6.70

6.80

6.30

6.50

6.50

7.30

6.70

7.00

6.70

7.10

-
-
6.0
5.0
4.0
6.0
6.0

5.0

4.0

6.0

6.0

8.2

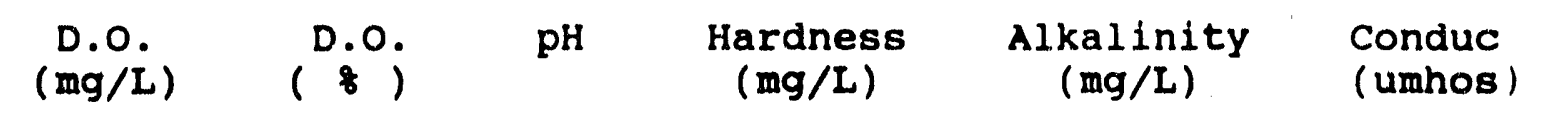

$100 \%$ Effluent

Initial

Day 1 (old)

Day 1 (new)

Day 2 (old)

Day 2 (new)

Day 3 (old)

Day 3 (new)

Day 4 (old)

Day 4 (new)

Day 5 (old)

Day 5 (new)

Day 6 (old)

Day 6 (new)
8.3

8.1

8.3

8.1

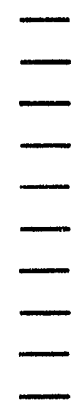

7.00

7.80

6.90

7.30

$<1.0$

23.2

200

1018

$<1.0$

24.2

221

$105 \% \quad 7.30$

79

84

57

55

38

35

34

Final

8.42

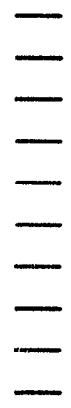

BIO - 871 
GOAIIIYY COYMROL

\section{Client: Savannah River site \\ Sample Identification: H-F Area (U3RC) \\ start Date: June 27, 1991}

Culture Health:

Sensitivity: Culture organisms were exposed to $\mathrm{NaCl}$ as a reference toxicant.

Current LC50: $\quad 0.707 \mathrm{~g} / \mathrm{L}$

Date: $7 / 25 / 91$

Reproduction: Control test organisms

Reproduction:

20.8 (young/female)

BIO - 871 


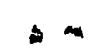
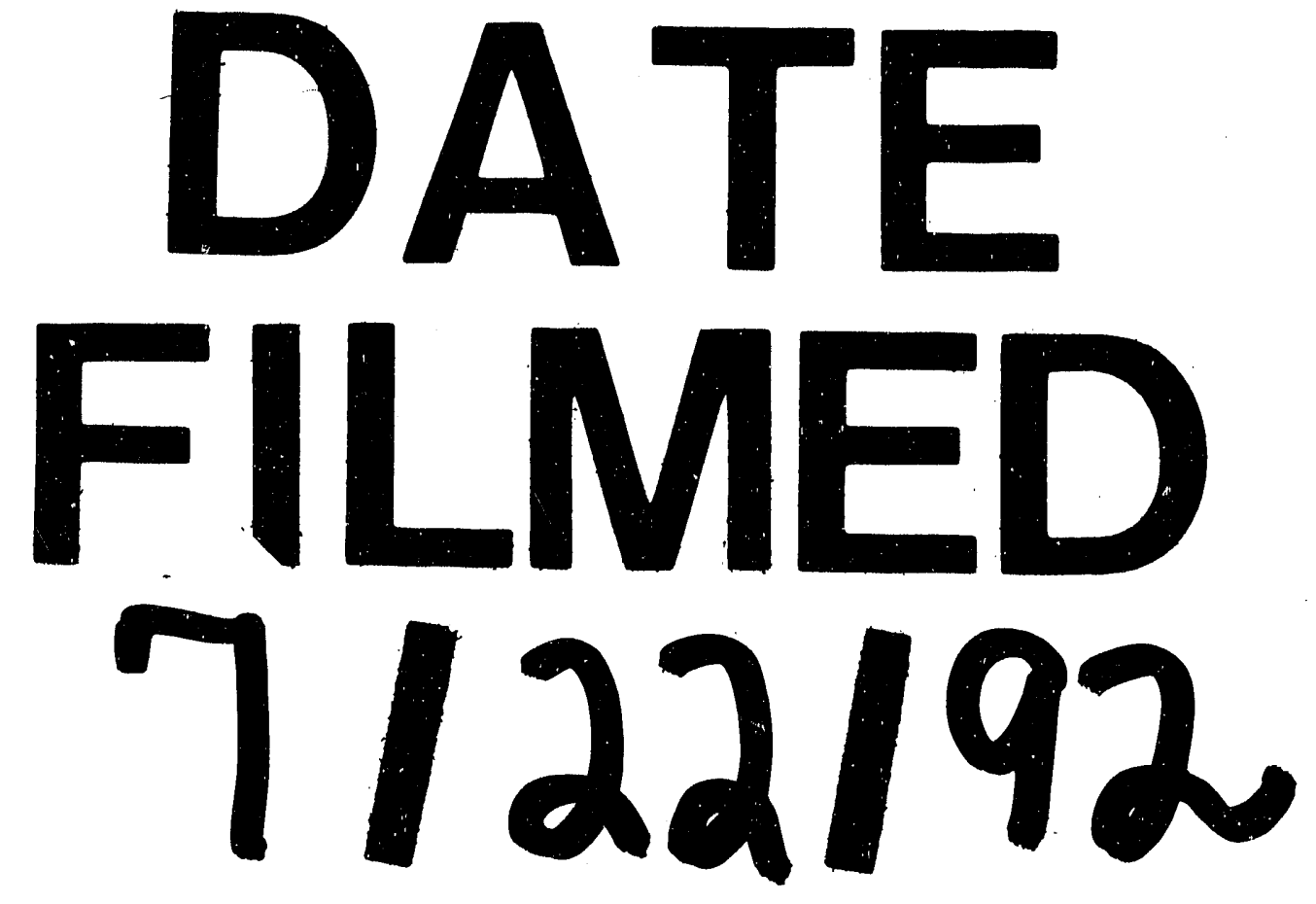

곡 
